\title{
Interação de atributos atrativos e obrigatórios de um serviço na satisfação do cliente
}

\author{
Gérson Tontini
}

André José Sant'Ana

FURB

\section{Resumo}

O Modelo Kano de Qualidade faz distinção entre três tipos de atributos que influenciam a satisfação do cliente: obrigatórios, atrativos e unidimensionais. Este trabalho teve como objetivo estudar a interação entre atributos atrativos e obrigatórios. Para tanto, utilizou-se um delineamento experimental do tipo fatorial completo com quatro atributos de video-locadoras (dois obrigatórios e dois atrativos), cada um a dois níveis, entrevistando-se 152 clientes. Verificou-se que os atributos atrativos apresentaram um maior impacto na satisfação dos clientes se os atributos obrigatórios estiverem presentes. A implicação gerencial desse resultado é que os atributos obrigatórios devem ser identificados e mantidos em um nível adequado, pois somente assim os atributos atrativos terão pleno efeito no aumento da satisfação dos clientes.

Palavras-chave

Modelo Kano, mensuração conjunta, satisfação do consumidor, qualidade em serviços, gestão da qualidade.

\section{Interaction of basic and excitement service attributes in customer satisfaction}

\begin{abstract}
Kano Model distinguishes service attributes in three types: basic, excitement and performance attributes. This work has as objective to verify how these attributes interact to proportionate satisfaction to customers. A full factorial experiment, using a sample of 152 customers of video rental stores, was used to study the interaction among four attributes (two basic and two excitements). The results show that the excitement attributes had less impact on customer satisfaction if the basic attributes were not fulfilled. The managerial implication of these results is that service companies should identify and fulfill the basic attributes in order to the excitement attributes having full impact on customer satisfaction.
\end{abstract}

Key words

Kano model, conjoint analysis, customer satisfaction, service quality, quality management. 


\section{INTRODUĈ̣̃O}

A satisfação dos clientes é considerada fator crítico para o sucesso das organizações em um mercado competitivo. Mas como uma empresa pode proporcionar satisfação de forma contínua aos seus clientes? A satisfação está relacionada com o atendimento de necessidades explícitas e implícitas do consumidor por meio do conjunto de características, ou atributos, do serviço. Então, torna-se importante descobrir como o desempenho dos diferentes atributos está relacionado com a satisfação dos clientes. Para tanto, tradicionalmente, tenta-se descobrir quais atributos podem satisfazer as necessidades dos clientes perguntando-se qual sua importância para o consumidor e o grau de desempenho atual de cada um em relação à concorrência. Esta técnica pressupõe que a relação entre o desempenho dos atributos e a satisfação do consumidor seja linear, isto é, quanto maior o desempenho maior a satisfação.

O Modelo Kano de qualidade atrativa e obrigatória (KANO et al., 1984) propõe que a relação entre desempenho e satisfação não é linear, classificando os atributos como obrigatórios (básicos), unidimensionais (performance) e atrativos (excitantes). Os atributos obrigatórios são aqueles considerados básicos ao serviço, cuja presença o cliente não notará e não trarão satisfação superior. Já sua ausência traz forte insatisfação. Atributos unidimensionais, também chamados de atributos de performance, são aqueles cuja satisfação é proporcional ao desempenho. Quanto maior o desempenho maior a satisfação e vice-versa. Atributos atrativos são aqueles cuja presença, ou desempenho superior, traz satisfação superior, porém sua ausência, ou desempenho inferior, não causa insatisfação.

Segundo Huiskonen e Pirttilä (1998), e Tontini e Silveira (2007), ignorar este relacionamento não linear entre o desempenho dos atributos e a satisfação geral do cliente pode levar a decisões errôneas na hora de se identificar quais atributos são críticos e quais ações devem ser tomadas para aumentar a satisfação dos clientes. Entre estas decisões está melhorar um atributo básico acima do nível médio do mercado. Atributos básicos não trarão satisfação superior se a empresa tiver desempenho acima dos concorrentes e acima da média do mercado. Neste caso, o esforço de melhoria seria desnecessário. Por outro lado, introduzir um atributo atrativo, de alto custo, por se estar com desempenho inferior aos concorrentes também pode ser um erro, pois um desempenho inferior nestes atributos não traz insatisfação aos clientes. Neste caso a empresa poderia estar desviando recursos de outras áreas mais relevantes.

Assim, a identificação da classificação dos atributos segundo o Modelo Kano e a utilização desta informação no gerenciamento da qualidade dos serviços tem sido alvo de diversos trabalhos publicados na literatura.

Matzler et al. (1996) e Tontini (2003) apresentam o Modelo Kano e explicam como utilizá-lo na identificação de atributos atrativos e obrigatórios de produtos e serviços. Huiskonen e Pirttilä (1998) discorrem sobre a aplicação do Modelo Kano aos atributos de serviços logísticos. O estudo sugere que este método pode ser usado para identificar os atributos mais importantes para os clientes e para a empresa.

\section{\satisfação do consumidor está necessidades explícitas e implícitas por meio dos atributos do serviço.}

Ting e Chen (2002), através de uma pesquisa de campo com 435 clientes e utilizando análise de regressão linear com variáveis dummy, classificaram 43 atributos de hipermercados em Taiwan de acordo com o Modelo Kano. Os autores concluíram que, dos atributos estudados, 17 podiam ser considerados como atrativos, 22 unidimensionais, 1 obrigatório e 3 neutros. Matzler, Fuchs e Schubert (2004), também usando análise de regressão com variáveis dummy, encontraram o relacionamento assimétrico entre a satisfação com diferentes fatores do trabalho e a satisfação geral dos empregados de uma empresa farmacêutica, confirmando o Modelo Kano no contexto de satisfação de empregados.

Wassenaar et al. (2003) usam o Modelo Kano para identificar uma hierarquia de atributos chaves para os consumidores de motores de veículos, melhorando, através desta identificação, a confiabilidade de modelos de previsão de demanda.

Tontini et al. (2004) utilizam uma metodologia modificada do Modelo Kano, integrando-a com a análise de importância e desempenho, proposta por Martilla e James (1977), para identificar a posição competitiva de 6 atributos de uma loja de material fotográfico.

Walter, Tontini e Domingues (2005) utilizam o Modelo Kano para analisar a classificação de 20 atributos e identificar oportunidades de melhoria em um curso superior. Em seu trabalho, os autores fazem uma comparação dos resultados apontados pela Matriz de Importância e Desempenho e pelo Modelo Kano, concluindo que a utilização conjunta dos dois métodos levou a uma melhor priorização dos atributos a serem melhorados. 
Zhang e Dran (2001) mostram a aplicação do Modelo Kano em websites e concluem que o modelo pode ser usado para auxiliar no controle da qualidade em termos das três classificações (atrativo, obrigatório, unidimensional) e para verificar variações na classificação dos atributos com o tempo. Concluem ainda que diferentes websites apresentam importâncias diferentes para os mesmos atributos, embora alguns sejam considerados altamente importantes em todos os 6 websites estudados. Também estudando websites, Cheung e Lee (2005), através de análise de regressão, estudaram o efeito não linear do desempenho de atributos na satisfação dos usuários. Segundo os autores, confiabilidade da informação on-line, acesso ao sistema e usabilidade tiveram um impacto maior na satisfação quando têm baixo desempenho que quando apresentam alto desempenho, podendo ser classificados como obrigatórios pelo Modelo Kano. Já utilidade e entendimento das informações e facilidade de navegação das páginas tiveram maior impacto quando seu desempenho é superior que inferior, sendo classificados como atrativos pelo Modelo Kano. de Tontini e Silveira (2007) e fazem uma aplicação de sua proposta na identificação de oportunidades de melhoria em videolocadoras.

Além de trabalhos de aplicação e de desenvolvimento teórico de métodos para identificação da classificação dos atributos segundo o Modelo Kano, poucos trabalhos têm se dedicado a verificar a dinâmica dos atributos com o tempo. Neste sentido, Nilsson-Witell e Fundin (2005) conduziram uma pesquisa sobre um serviço on-line de venda de ingressos para cinema, procurando identificar a consistência e dinâmica da teoria do Modelo Kano. Os resultados mostram que quando o serviço on-line foi introduzido, era percebido como indiferente pelos clientes. No tempo da pesquisa, o serviço parecia ser considerado atrativo pelo mercado, porém, os primeiros usuários do serviço já o percebiam como unidimensional ou obrigatório. Esses resultados sugerem que a classificação dos atributos é dinâmica e varia de acordo com o quão novo é o atributo, com o costume dos clientes e com a oferta dos competidores.

Nenhum trabalho foi encontrado na literatura que procure identificar como atributos atrativos, obrigatórios e unidimensionais interagem para trazer satisfação aos clientes. Os métodos empregados nos estudos anteriores não levam em consideração a interação dos atributos entre si e a influência dessa interação na satisfação dos clientes. O estudo dessas interações é importante porque pode levar ao desenvolvimento de métodos mais refinados para o projeto de produtos e serviços, que apontem não apenas quais

Löfgren e Witell (2005) fazem um estudo da aplicação do Modelo Kano em embalagens, procurando identificar como seus diferentes atributos afetam a decisão de compra dos consumidores. Para tanto, 24 atributos foram investigados entrevistando-se 1500 consumidores de produtos de uso diário. Os autores encontraram evidências da existência de diferentes tipos de atributos, sendo que alguns, como material reciclável e a possibilidade de as embalagens serem seladas novamente após o uso, podem ser considerados como atrativos, influenciando a decisão de compra e uso do produto.

Alguns trabalhos têm estudado o Modelo Kano do ponto de vista teórico. Picolo (2005) faz uma comparação de diversos métodos para identificar oportunidades de melhoria em serviços e para classificar os atributos segundo o Modelo Kano. Tontini e Silveira (2007) fazem uma análise crítica do Modelo Kano tradicional e da análise por meio da Matriz de Importância e Desempenho na identificação de oportunidades de melhoria, propondo que esta identificação seja feita pela análise competitiva dos gaps de melhoria. Tontini e Sant'Ana (2007) sugerem modificações no método atributos são relevantes para a satisfação dos clientes, mas que também ajudem a determinar uma combinação ótima entre eles. Então, o presente trabalho tem como objetivo estudar como atributos atrativos e obrigatórios interagem para trazer satisfação aos clientes.

$\mathrm{O}$ artigo está estruturado da seguinte forma: a próxima seção apresenta os procedimentos metodológicos usados no trabalho. A seção "Identificação dos atributos segundo o Modelo Kano" utiliza uma modificação no método proposto por Tontini e Sant'Ana (2007) para fazer a identificação dos atributos a serem utilizados neste estudo. Embora qualquer produto ou serviço pudesse ser objeto de estudo, o serviço "videolocadoras" foi utilizado por ser de conhecimento geral dos respondentes e por já ter sido utilizado em pesquisa anterior, o que possibilitou a escolha efeito da interação dos atributos atrativos e obrigatórios" faz a análise dos resultados da pesquisa, identificando como os atributos interagem na formação da satisfação dos clientes. A seção "Considerações finais" apresenta as conclusões do estudo. dos atributos estudados nesta pesquisa. A seção "Estudo do 


\section{MATERIAIS E MÉTODO}

Este trabalho foi realizado em duas etapas. Em uma primeira etapa, os dados da pesquisa realizada por Tontini e Sant'Ana (2007) foram utilizados para classificar os atributos segundo o Modelo Kano e identificar quatro atributos de videolocadoras, sendo dois atrativos (Caixa Coletora e Programa Fidelidade) e dois obrigatórios (Cortesia no atendimento e Variedade de Filmes).

Segundo Montgomery (2004, p. 370), quando há vários fatores de interesse e se quer verificar a contribuição e a interação entre os fatores, o planejamento experimental deve ser utilizado. Assim, a interação entre os quatro atributos identificados na primeira fase foi estudada por meio de um planejamento experimental do tipo fatorial completo com réplicas, com os quatro atributos variando a dois níveis cada, totalizando $\mathrm{N}=2 \mathrm{k}=24=16$ combinações.

A população pesquisada é composta pelos alunos de um curso de graduação em administração, noturno, totalizando 468 alunos. O tamanho da amostra foi determinado pelos alunos presentes na sala de aula no momento da aplicação do questionário e que aceitaram participar da pesquisa. Assim, após a aplicação da pesquisa e a eliminação de questionários inválidos, obteve-se uma amostra de 152 respondentes, ou $32 \%$ da população. Foram considerados inválidos questionários com excessivo número de respostas em branco ou que não apresentavam variação nas respostas. Apesar de não ser uma amostra aleatória estratificada e proporcional, como os alunos provêm de diferentes regiões e classes sociais, e freqüentam videolocadoras diferentes, considerou-se esta amostra como adequada para a realização do trabalho.

O questionário de coleta de dados foi composto de três partes. Na primeira parte, os respondentes tiveram que assinalar seu grau de satisfação com as 16 diferentes combinações dos atributos pesquisados (Caixa Coletora, Variedade de Filmes, Programa Fidelidade e Cortesia e Gentileza no atendimento) (Anexo 1). O efeito da presença de cada atributo na satisfação dos clientes é calculado pela diferença entre a média da satisfação quando os atributos estão presentes (nível alto) e a média da satisfação apontada pelos respondentes quando os atributos estão ausentes (nível baixo). Isto é, por exemplo, o efeito da presença da Caixa Coletora na satisfação geral é dado pela diferença entre a média dos resultados das linhas 5, 7, 8, 9, 10, 12, 15 e 16 do questionário (Anexo 1) e a média dos resultados das linhas $1,2,3,4,5,11,13,14$, entre todos os respondentes (média das linhas e réplicas). Segundo Hair (2005), esta técnica é baseada na premissa simples de que os consumidores avaliam o valor do produto ou serviço pela combinação do valor dos diferentes atributos. Uma vantagem deste método, chamado de mensuração conjunta pelos praticantes de marketing (GREEN; KRIEGER; WIND, 2001), é que ele mede o efeito dos atributos com o respondente fazendo uma negociação entre eles, pensando na combinação dos mesmos e não isoladamente. Segundo Gibson (2001), uma desvantagem é que o esforço para responder ao questionário é maior e o número de variáveis a serem pesquisadas é menor que nas avaliações individuais.

Para garantir que a distribuição dos dados se aproximasse da normal, os respondentes foram agrupados aleatoriamente em amostras de tamanho cinco. A média da satisfação apontada pelos respondentes de cada amostra foi utilizada para análise dos dados. Assim, o tamanho final da amostra foi de $152 / 5 \approx 30$. O efeito dos atributos e suas interações foram testados utilizando-se o teste $t$ para diferenças entre as médias com amostras pareadas.

\section{A classificação dos atributos segundo o Modelo Kano varia com a novidade do atributo, com o costume dos clientes e com a oferta dos competidores.}

Na segunda parte do questionário, pediu-se aos respondentes para assinalar, em uma escala de 0 a 10 , sua avaliação sobre o desempenho atual dos atributos pesquisados na videolocadora que eles mais freqüentam e a sua satisfação geral com aquela videolocadora. Na terceira parte pediu-se aos respondentes que explicitassem qual a videolocadora que mais freqüentam, alguns dados demográficos e se os atributos pesquisados (Programa Fidelidade e Caixa Coletora) estão presentes na videolocadora que mais freqüentam.

\section{RESULTADOS E DISCUSSÃO}

\section{Identificação dos atributos segundo o Modelo Kano}

Kano et al. (1984), Berger et al. (1993) e Matzler et al. (1996) sugerem que os atributos obrigatórios, unidimensionais e atrativos podem ser identificados perguntando-se aos consumidores sobre sua reação, ou satisfação, quando os atributos têm desempenho superior (questão positiva) e inferior (questão negativa). Dependendo das respostas para as questões "positivas" e "negativas", o atributo pode ser identificado como atrativo, obrigatório, unidimensional, neutro ou reverso. Segundo Tontini e Silveira (2007), apesar do modelo Kano trazer uma visão diferente sobre o efeito do desempenho dos atributos na satisfação do consumidor, 
ele não leva em consideração o nível atual de desempenho dos atributos. Como a posição competitiva não é levada em consideração, o modelo Kano tradicional, usado de maneira isolada, é limitado como um direcionador dos esforços de melhoria.

Assim, para direcionar os esforços de melhoria, Tontini e Silveira (2007) propõem a classificação dos atributos segundo o Modelo Kano por meio do cálculo de gaps. No método proposto por esses autores, pede-se aos clientes para assinalarem seu grau de satisfação em três situações: se o atributo tiver desempenho superior (Kano positivo), inferior (Kano negativo) e a satisfação atual com o desempenho do atributo. Então, os gaps de satisfação com o aumento (Kano positivosatisfação atual) ou diminuição (satisfação atual - Kano negativo) no desempenho dos atributos são calculados. Os atributos são classificados como atrativos se o gap positivo for significativo e o gap negativo for não significativo. São classificados como unidimensionais quando ambos os gaps são significativos e obrigatórios quando o gap positivo não é significativo e o gap negativo for significativo.

Tontini e Sant'Ana (2007), fazendo uma aplicação do método desenvolvido por Tontini e Silveira (2007) na classificação dos atributos e identificação de oportunidades de melhoria em videolocadoras, identificaram que os gaps negativos tendem a ter um valor absoluto maior que os gaps positivos, como pode ser visto na Figura 1.

Segundo esses autores, o consumidor geralmente tem algum nível de satisfação média positiva com o desempenho atual dos atributos e tende a avaliar as situações negativas de maneira extrema. Assim, no método proposto por Tontini e Silveira (2007), "existe a tendência de o gap negativo ser maior que o gap positivo" (TONTINI; SANT'ANA, 2007, p. 50). Este viés pode levar a um aumento na identificação de atributos classificados como unidimensionais ou obrigatórios, mascarando a identificação de atributos atrativos.

Procurando corrigir este problema, Tontini e Santa `Ana (2007) propõem uma alteração no método de Tontini e Silveira (2007) para identificar a classificação de dezenove atributos de videolocadoras segundo o Modelo Kano. No método proposto, o gap positivo é dado pelo incremento de satisfação que a melhoria (ou presença) do atributo trará em relação a média atual (Kano Positivo - Média atual), e o "gap" negativo é dado pelo valor absoluto de insatisfação gerada pela ausência ou insuficiência do atributo. Os

Figura 1: Análise dos Gaps de melhoria para as Videolocadoras.

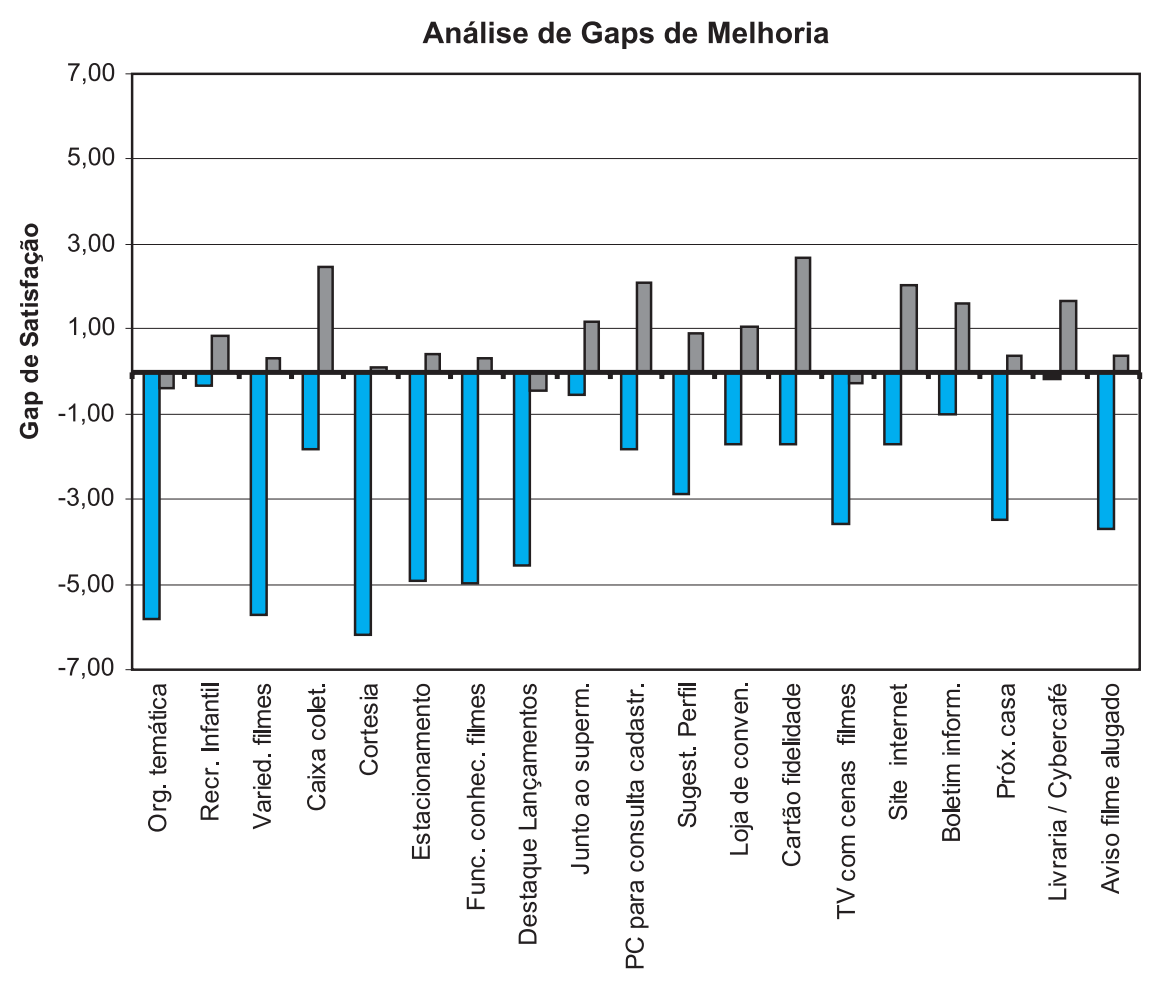

Fonte: Tontini e Sant'Ana, 2007. 
atributos são considerados significativos como potenciais provedores de satisfação ou insatisfação se os gaps positivo e negativo ultrapassarem as linhas de neutralidade, estabelecidas em $\pm 1,5$ por Tontini e Sant’Ana (2007). Isto é, atributos que apresentam incremento na satisfação maior que 1,5 são considerados relevantes para melhoria ou oferta. Já atributos que trazem insatisfação média maior que $-1,5$ com a sua ausência são considerados significativos como possíveis geradores de insatisfação geral.

Segundo Tontini e Sant'Ana (2007, p. 50), “não há regra específica para a definição do valor das linhas divisórias de neutralidade, porém o raciocínio por traz deste valor $( \pm 1,5)$ é que o incremento de satisfação com a melhora tem que ser maior que, por exemplo, passar de levemente satisfeito para satisfeito. Já a ausência do atributo tem que gerar uma insatisfação maior que levemente insatisfeito para ser considerada significativa". A definição do gap - como sendo o valor absoluto de insatisfação gerada pelo baixo desempenho do atributo e o posicionamento das linhas de neutralidade dos gaps em $\pm 1,5$ é uma sistemática empírica que ainda deve ser objeto de estudos para sua validação.

Uma alternativa a esse método é calcular os gaps positivo e negativo segundo o método apresentado por Tontini e Silveira (2007) e efetuar sua padronização, dividindo-os pelo desvio padrão dos gaps (positivos ou negativos) de todos os atributos estudados. Assim, o viés de os gaps negativos serem maiores em média que os gaps positivos é eliminado. As linhas divisórias de neutralidade são es- tabelecidas pela média dos gaps padronizados positivos e negativos. Esta alternativa apresenta a vantagem de eliminar o viés dos gaps negativos por um método estatístico, porém ele é dependente dos atributos pesquisados. A Figura 2 mostra o resultado da classificação dos atributos das videolocadoras estudados por Tontini e Sant Ana (2007) usando os gaps padronizados.

Analisando-se a Figura 2 verifica-se que os atributos Caixa Coletora e Cartão Fidelidade são os que apresentam maior ganho de satisfação caso estejam presentes, sendo considerados atrativos, pois não trazem insatisfação significativa se ausentes. Já Cortesia, Variedade de Filmes e Organização Temática são os atributos que trazem maior insatisfação se estiverem ausentes, sendo classificados como obrigatórios, pois não trazem satisfação significativa se presentes. Os resultados mostrados na Figura 2 encorajam a utilização dos gaps padronizados, pois os atributos que pelo bom senso seriam atrativos assim aparecem. Já aqueles que o bom senso diz serem obrigatórios, também são classificados como tais. Assim, escolheram-se para este estudo os atributos Caixa Coletora e Programa Fidelidade como atrativos, e Cortesia e Variedade de Filmes como obrigatórios.

\section{Estudo do Efeito da Interação dos atributos Atrativos e Obrigatórios}

A Tabela 1 mostra o efeito médio da ausência ou presença de cada atributo na alteração da satisfação apontada pelos respondentes da pesquisa, utilizando o questionário com

Figura 2: Classificação dos Atributos pela Análise de gaps Padronizados.

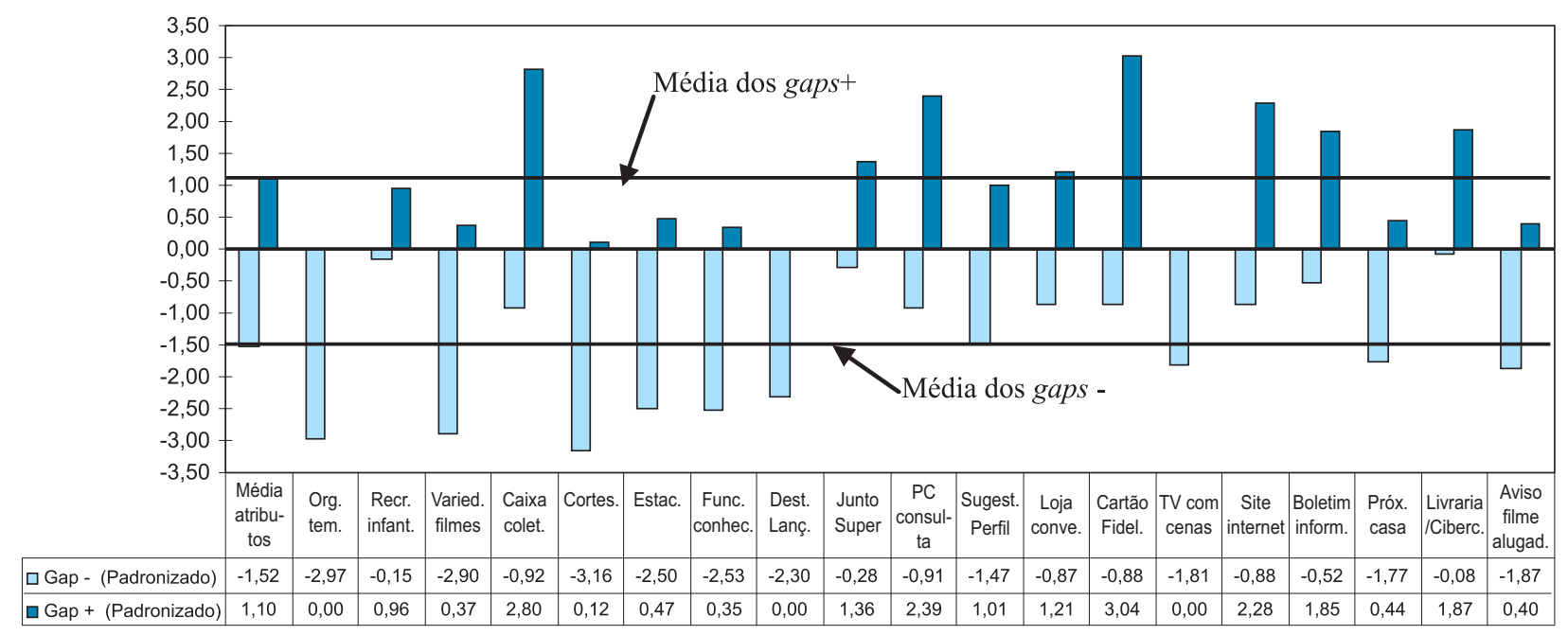

Fonte: autores. 
delineamento experimental mostrado no Anexo 1. A variação na satisfação, apontada pelos respondentes quando um atributo passa a estar presente, é utilizada para se identificar sua importância relativa. Assim, analisando a última coluna da Tabela 1, verifica-se que Cortesia é três vezes mais importante que o Programa Fidelidade. Os atributos considerados atrativos (Caixa Coletora e Programa Fidelidade) são menos importantes, na média, que os atributos considerados obrigatórios (Variedade de Filmes e Cortesia). Estes resultados confirmam, em parte, o apontado por Sauerwein (1999) de que a importância atribuída pelos clientes aos atributos é decrescente nesta ordem: obrigatórios $=>$ unidimensionais $\Rightarrow$ atrativos $=>$ neutros.

A figura 3B mostra a interação entre os atributos Variedade, Cortesia e Programa Fidelidade. A linha sólida mostra que o ganho de satisfação obtido pela introdução do Programa Fidelidade, quando Variedade e Cortesia estão em nível insuficiente, é de 0,85 ( $\mathrm{p}$-value $=0,000)$. Porém, quando Variedade e Cortesia estão em nível suficiente (linha pontilhada), esse ganho de satisfação passa para 1,47 (p-value $=$ $0,000)$. Esta diferença de ganho de satisfação foi considerada significante no teste t para diferenças das médias com amostras pareadas $(\mathrm{p}$-value $=0,003)$.

As Figuras 3A e 3B indicam a existência de interação entre os atributos atrativos e os obrigatórios, sendo que o efeito da presença dos atributos atrativos é praticamente duplicado quando os atributos obrigatórios estão presentes ou em nível suficiente. É necessário verificar ainda a existência de interação entre os atributos atrativos, independentemente da presença ou não de atributos obrigatórios.

A Figura 3C mostra a interação entre os atributos Caixa Coletora e Programa Fidelidade, ambos atributos atrativos. A linha sólida mostra

O objetivo da pesquisa é verificar como os atributos interagem para trazer satisfação ao consumidor. Para tanto, procurou-se investigar os efeitos das interações entre os atributos atrativos e obrigatórios através de uma análise gráfica (Figura 3).

A Figura 3A mostra a interação entre os atributos Variedade, Cortesia e Caixa Coletora. A linha sólida mostra que o ganho de satisfação obtido pela existência da Caixa Coletora, quando Variedade e Cortesia estão em nível insuficiente, é de 0,59. Este ganho foi considerado significante no teste $t$ para diferenças das médias com amostras pareadas $(\mathrm{p}$-value $=0,000)$. Porém, quando Variedade e Cortesia estão em nível suficiente (linha pontilhada), esse ganho de satisfação passa para 1,38 ( $\mathrm{p}$-value $=0,000$; teste $\mathrm{t}$ com amostras pareadas). Esta diferença no ganho de satisfação (de 0,59 para 1,38) foi considerada significante no teste $t$ para diferenças das médias com amostras pareadas $(p$-value $=0,000)$. que o ganho de satisfação obtido pela existência de Programa Fidelidade, quando o atributo Caixa Coletora está ausente, é de 0,79 ( $\mathrm{p}$-value $=0,000$ ). Porém, quando Caixa Coletora está presente (linha pontilhada), esse ganho de satisfação passa para $1,15$ ( $p$-value $=0,000)$. Esta diferença de ganho de satisfação foi considerada significante no teste $t$ para diferenças das médias com amostras pareadas ( $p$-value $=0,028)$. Este resultado indica a existência de interação entre os atributos atrativos.

A Figura 3D mostra a interação entre os atributos Variedade, Cortesia, Caixa Coletora e Programa Fidelidade. A linha sólida mostra que o ganho de satisfação obtido pela introdução conjunta da Caixa Coletora e Programa Fidelidade, quando Variedade e Cortesia estão em nível insuficiente, é de $1,43(p$-value $=0,000)$. Porém, quando Variedade e Cortesia estão em nível suficiente (linha pontilhada), esse ganho de satisfação passa para 2,85 (p-value $=0,000)$. Esta diferença de ganho de satisfação foi considerada significante no teste $t$ para diferenças das médias com amostras pareadas ( $\mathrm{p}$-value

Tabela 1: Efeito da Presença ou Ausência dos Atributos na Satisfação.

\begin{tabular}{|l|c|c|c|c|}
\hline \multicolumn{2}{|c|}{ SATISFAçÃO MÉDIA } & GANHO SAT. & P-VALUE \\
\hline Variedade & - & + & 2,25 & 0,000 \\
\hline Cortesia & $-1,57$ & 0,68 & 2,94 & 0,000 \\
\hline Fidelidade & $-1,91$ & 1,02 & 0,97 & 0,000 \\
\hline Caixa & $-0,93$ & 0,04 & 1,04 & 0,000 \\
\hline Fonte: dados da pesquisa & $-0,96$ & 0,07 & & . \\
\hline
\end{tabular}


$=0,000)$. Este resultado mostra que o efeito do conjunto dos atributos atrativos (Caixa Coletora e Programa Fidelidade) tem seu efeito na satisfação dobrado quando os atributos obrigatórios estão presentes.

Apesar de não constar na Figura 3, estudou-se também a interação entre os dois atributos obrigatórios (Cortesia e Variedade). O cálculo do efeito das interações mostra que, quando Cortesia está ausente, a suficiência de variedade tem um efeito de 1,81 na satisfação $(p$-value $=0,000)$. Já quando Cortesia está presente, a variedade tem um efeito de 2,70 na satisfação geral ( $p$-value $=0,000$ ). Esta diferença de ganho na satisfação foi considerada significante no teste $t$ para diferenças nas médias com amostras pareadas ( $p$-value $=0,000)$. Este resultado indica que os atributos obrigatórios também apresentaram interação entre si.
O resultado das análises das interações, efetuadas nos parágrafos anteriores, mostra uma intensa interação entre todos os atributos, indicando que o efeito dos atributos na satisfação do consumidor é complexo, não podendo ser estimado apenas por um modelo aditivo simples. Assim, para estimar o efeito da presença ou ausência dos atributos individuais na satisfação geral, ajustou-se uma equação de regressão entre a satisfação assinalada pelos respondentes (variável dependente) e os atributos usados no delineamento experimental como variáveis dummy independentes, sendo estabelecido o valor 1 para a existência da variável e 0 para a inexistência. Havendo 16 respostas para cada respondente e 152 respondentes, com a satisfação média sendo calculada de cinco em cinco respondentes, a análise de regressão utilizou 480 casos. Os dados perdidos foram eliminados pelo

Figura 3: Gráficos dos efeitos das interações entre os atributos.

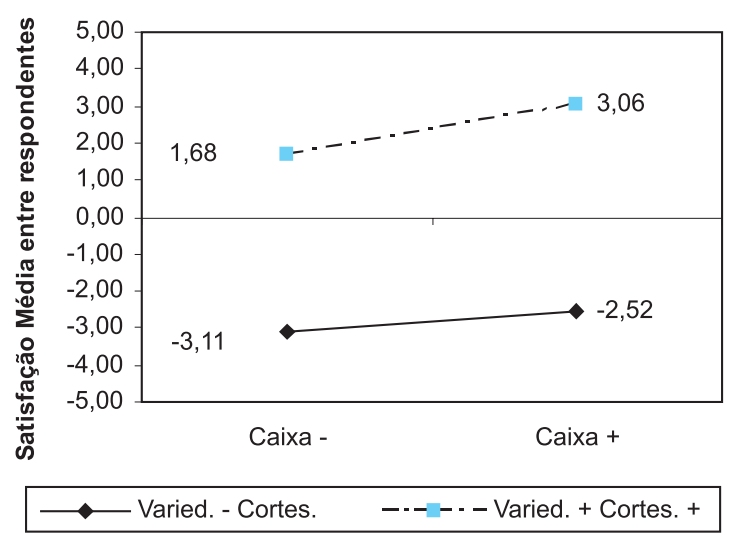

Fig. 3A - Interação Variedade-Cortesia x Caixa

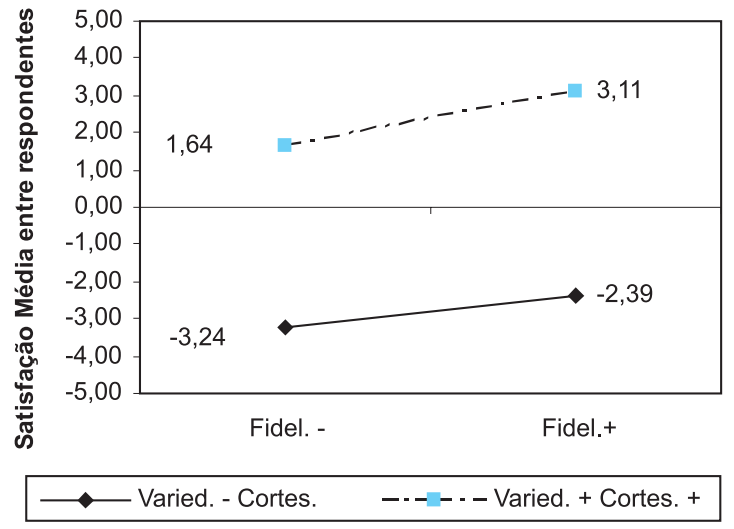

Fig. 3B - Interação Variedade-Cortesia x Fidelidade

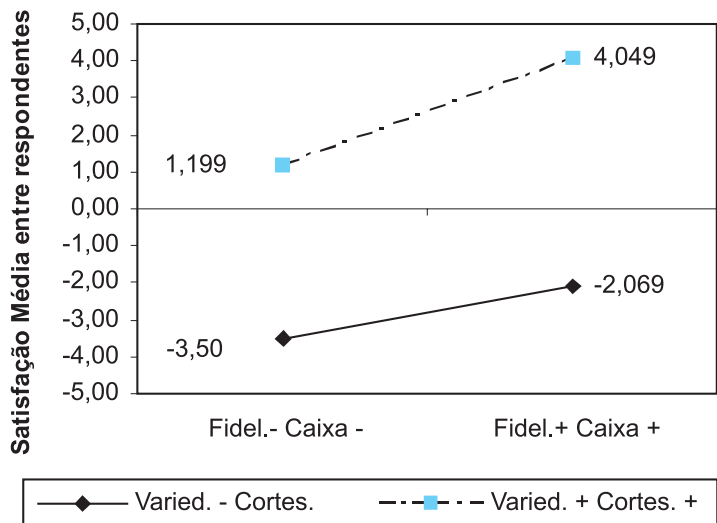

Fig. 3D - Interação Variedade-Cortesia x Fidel. -Caixa

Fig. 3C - Interação Caixa x Fidelidade 
método "listwise"do SPSS, totalizando 445 casos válidos.

Como diversas equações de regressão são possíveis entre as variáveis independentes e suas interações, e a variável dependente, utilizou-se o método de regressão tipo "Backward", incluindo-se inicialmente os quatro atributos estudados, a interação entre os dois atributos obrigatórios (Variedade e Cortesia), entre os dois atrativos (Programa Fidelidade e Caixa Coletora) e entre os atributos obrigatórios e atrativos (Prog. Fidelidade x Cortesia x Variedade e Caixa Coletora x Cortesia x Variedade). A Tabela 2 mostra os resultados para o ajuste da equação utilizando o método Backward. O coeficiente de determinação ajustado ( $\mathrm{R}^{2}$ ajustado) ficou em 0,736 , mostrando que a equação de regressão apresenta um bom poder de explicação da satisfação média esperada entre os respondentes.

A Figura 4 mostra o histograma e o gráfico de dispersão dos resíduos padronizados da regressão. De acordo com o teste de Kolmorov-Smirnov (validade assintótica = 0,270) se aceita a hipótese de que os resíduos seguem a distribuição normal, indicando, portanto, que a equação de regressão é válida.

Analisando a Tabela 2 verifica-se que a interação entre os atributos Variedade e Cortesia não foi significativa ( $\mathrm{p}$-value $=0,288$ ), tendo sido excluída do modelo. Esta falta de significância contraria a análise efetuada por meio do cálculo das interações da Figura 3. Esta divergência de resultados pode ser devida ao fato de que, na análise de regressão, a interação entre estes dois atributos teve que competir com as outras interações para entrar na equação, ao contrário do cálculo da interação média feita pela análise experimental. Isto pode ser uma indicação de que os atributos obrigatórios são aditivos entre si. A interação entre os atributos atrativos também foi considerada não significante ( $p$-value $=0,135)$, indicando que os atributos atrativos podem ser aditivos entre si. Por outro lado, os atributos obrigatórios (Cortesia e Variedade) tiveram interação com os atributos atrativos (Programa Fidelidade e Caixa Coletora), indicando que o efeito destes atributos é potencializado quando os dois primeiros encontram-se em nível satisfatório.

A Figura 5 mostra uma simulação da satisfação média prevista com a evolução da presença dos atributos, utilizando a equação de regressão mostrada na Tabela 2. A primeira coluna mostra uma numeração para a combinação dos atributos. As outras quatro colunas da esquerda da Figura 5 apresentam a situação de presença ou ausência dos atributos, onde " 1 " significa que o atributo está presente e " 0 " que o atributo está ausente. Cada linha representa uma combinação do conjunto de atributos. A sexta coluna da Figura 5 apresenta a satisfação prevista com a combinação dos atributos mostrada nas quatro colunas da esquerda. A sétima

Tabela 2: Equação de Regressão entre a Satisfação e os atributos pesquisados.

\begin{tabular}{|c|c|c|c|c|c|}
\hline \multirow[b]{3}{*}{ VARIÁVEIS NO MODELO } & \multicolumn{5}{|c|}{ GOEFIGIENTES } \\
\hline & \multicolumn{2}{|c|}{ COEF. NÃO PADRONIZ. } & \multirow{2}{*}{$\begin{array}{c}\text { COEF. PADRON. } \\
\text { BETA }\end{array}$} & \multirow[t]{2}{*}{ VALOR EST. $t$} & \multirow[t]{2}{*}{ P-VALUE } \\
\hline & $\mathbf{B}$ & ERRO PADRÃO & & & \\
\hline Constante & $-3,733$ & 0,147 & & $-25,427$ & 0,000 \\
\hline Variedade & 1,974 & 0,136 & 0,42 & 14,546 & 0,000 \\
\hline Cortesia & 2,649 & 0,135 & 0,564 & 19,603 & 0,000 \\
\hline Fidelidade & 0,775 & 0,129 & 0,165 & 6,014 & 0,000 \\
\hline Caixa Colet. & 0,922 & 0,129 & 0,196 & 7,15 & 0,000 \\
\hline $\begin{array}{l}\text { Cortesia x Variedade } \mathrm{x} \\
\text { Fidel. }\end{array}$ & 0,836 & 0,237 & 0,117 & 3,534 & 0,000 \\
\hline $\begin{array}{l}\text { Cortesia x Variedade x } \\
\text { Caixa }\end{array}$ & 0,444 & 0,237 & 0,062 & 1,874 & 0,062 \\
\hline \multicolumn{6}{|l|}{ Variáveis Eliminadas } \\
\hline $\begin{array}{l}\text { Variedade } \times \text { Cortesia } \\
\text { Fidelidade } \times \text { Caixa Colet. }\end{array}$ & & & & $\begin{array}{l}1,062 \\
1,497\end{array}$ & $\begin{array}{l}0,288 \\
0,135\end{array}$ \\
\hline
\end{tabular}

Método: "Backward regression"

$\mathrm{R}^{2}$ ajustado $=0,736$

$N=445$

Probabilidade F-remove $=0,10$

Fonte: Dados da pesquisa. 
coluna mostra o ganho de satisfação quando os atributos são introduzidos (de uma linha para a outra da tabela). Os gráficos representam os dados da satisfação média prevista (sexta coluna).
Nos dados referentes ao primeiro gráfico da Figura 5, os atributos são introduzidos na seguinte ordem: nenhum atributo $=>$ Cortesia $=>$ Variedade $=>$ Programa Fidelidade $=>$ Caixa Coletora. Verifica-se que a presença exclusiva de

Figura 4: Histograma e Dispersão dos Resíduos Padronizados da Regressão.

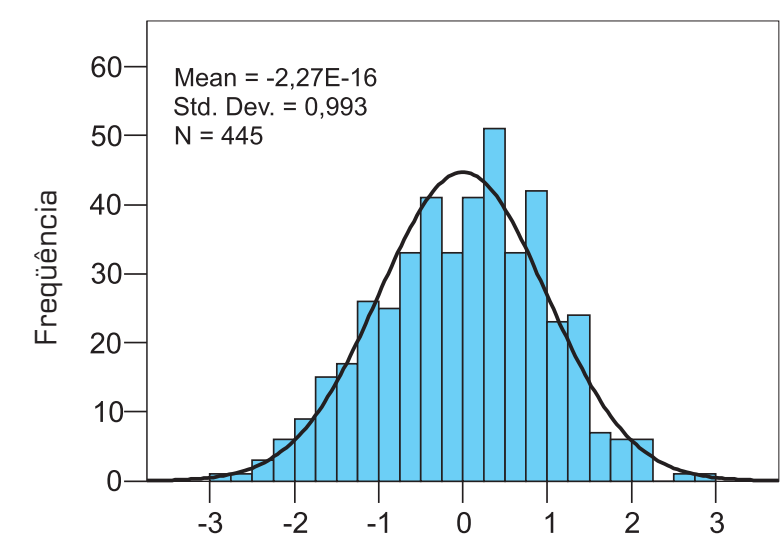

Resíduos Padronizados da Regressão

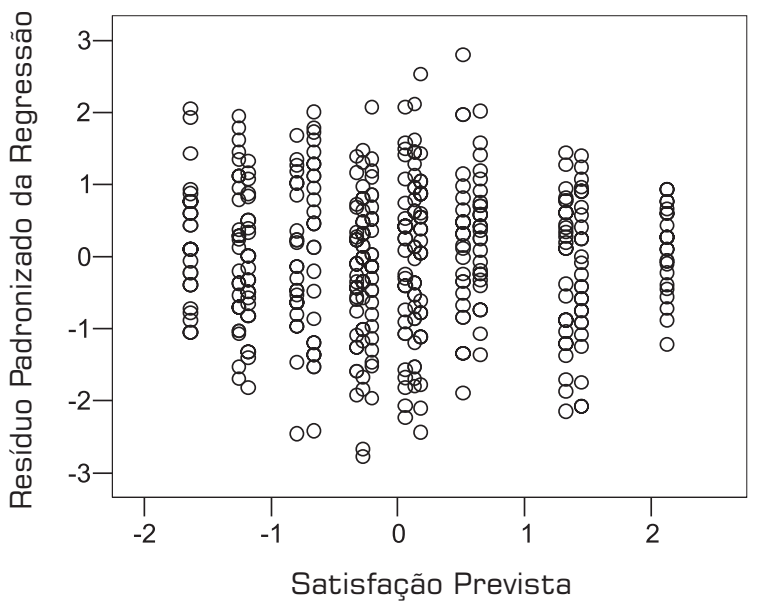

Fonte: Dados da pesquisa.

Figura 5: Evolução da Satisfação Prevista pela Presença dos Atributos.

\begin{tabular}{|c|c|c|c|c|c|c|c|c|}
\hline SIT. & CORTESIA & VARIEDADE & FIDELIDADE & $\begin{array}{l}\text { CAIXA } \\
\text { COL }\end{array}$ & $\begin{array}{l}\text { SATISF. } \\
\text { PREVISTA }\end{array}$ & $\begin{array}{l}\text { GANHO } \\
\text { SAT. }\end{array}$ & & $\begin{array}{l}\text { EVOLUÇÃO DA } \\
\text { SATISFAÇÃO }\end{array}$ \\
\hline 1 & 0 & 0 & 0 & 0 & $-3,73$ & & \multirow{5}{*}{$\begin{array}{r}4,00 \\
2,00 \\
0,00 \\
-2,00 \\
-4,00 \\
-6,00\end{array}$} & \multirow{5}{*}{ 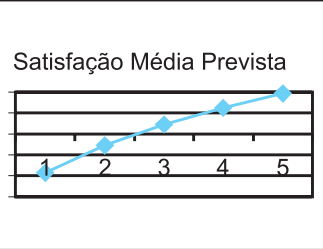 } \\
\hline 2 & 1 & 0 & 0 & 0 & $-1,08$ & 2,65 & & \\
\hline 3 & 1 & 1 & 0 & 0 & 0,89 & 1,97 & & \\
\hline 4 & 1 & 1 & 1 & 0 & 2,50 & 1,61 & & \\
\hline 5 & 1 & 1 & 1 & 1 & 3,87 & 1,37 & & \\
\hline 1 & 0 & 0 & 0 & 0 & $-3,73$ & & \multirow{5}{*}{$\begin{array}{r}4,00 \\
2,00 \\
0,00 \\
-2,00 \\
-4,00\end{array}$} & \multirow[b]{2}{*}{ Satisfação Média Prevista } \\
\hline 2 & 0 & 0 & 1 & 0 & $-2,96$ & 0,78 & & \\
\hline 3 & 0 & 0 & 1 & 1 & $-2,04$ & 0,92 & & 3 \\
\hline 4 & 0 & 1 & 1 & 1 & $-0,06$ & 1,97 & & $12=34$ \\
\hline 5 & 1 & 1 & 1 & 1 & 3,87 & 3,93 & & \\
\hline 1 & 0 & 0 & 0 & 0 & $-3,73$ & & \multirow{5}{*}{$\left.\begin{array}{r}4,00 \\
2,00 \\
0,00 \\
-2,00 \\
-4,00\end{array}\right]$} & \multirow{2}{*}{ Satisfação Média Prevista } \\
\hline 2 & 1 & 0 & 0 & 0 & $-1,08$ & 2,65 & & \\
\hline 3 & 1 & 0 & 0 & 1 & $-0,16$ & 0,92 & & $+2 r^{2}$ \\
\hline 4 & 1 & 0 & 1 & 1 & 0,61 & 0,78 & & 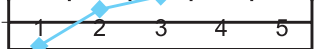 \\
\hline 5 & 1 & 1 & 1 & 1 & 3,87 & 3,25 & & \\
\hline
\end{tabular}

Fonte: dados da pesquisa. 
Cortesia e Variedade, atributos obrigatórios, traz uma satisfação prevista de 0,89 , levemente acima da neutralidade. Os ganhos de satisfação com a introdução desses atributos ficam em 2,65 e 1,97, respectivamente. Já a introdução dos atributos atrativos (Programa Fidelidade e Caixa Coletora) traz um ganho de 1,61 e 1,37, respectivamente, quando os atributos obrigatórios estão presentes. diversos atributos, sendo que um projeto superior, que inclua os atributos relevantes para o consumidor, pode trazer grande diferencial no mercado para a empresa. Assim, a identificação da relação entre o desempenho dos atributos e a satisfação do consumidor torna-se chave para o sucesso em um mercado competitivo.

O Modelo Kano de qualidade atrativa e obrigatória (KANO et al., 1984; BERGER et al., 1993; MATZLER et al., 1996) pressupõe a existência de uma relação não linear entre desempenho e satisfação, classificando os atributos como obrigatórios, unidimensionais e atrativos, dependendo de como eles influenciam a satisfação dos clientes. Segundo Kano et al.(1984), atributos classificados como atrativos podem trazer um diferencial no mercado. Por outro lado, ter desempenho superior em atributos obrigatórios não traz satis-

O segundo gráfico da Figura 5 mostra a evolução da satisfação quando os atributos são introduzidos na seguinte ordem: nenhum atributo $=>$ Programa Fidelidade $=>$ Caixa Coletora $=>$ Variedade $=>$ Cortesia. Neste caso verificase que o ganho de satisfação trazido pela introdução do Programa Fidelidade e Caixa Coletora é 0,78 e 0,92, respectivamente. Este ganho é cerca de $50 \%$ a $70 \%$ do ganho trazido pela introdução desses atributos quando Cortesia e Variedade estão presentes. Esses resultados demonstram que os atributos atrativos têm seu efeito na satisfação ampliado caso os atributos obrigatórios estejam presentes ou tenham desempenho suficiente. Ainda, verifica-se que a presença de Variedade, Programa Fidelidade e Caixa Coletora não é suficiente para compensar a falta da cortesia e trazer satisfação aos respondentes (satisfação prevista de -0,06).

O terceiro gráfico da Figura 5 mostra a evolução da satisfação com a introdução dos atributos na seguinte ordem: nenhum atributo $=>$ Cortesia $=>$ Caixa Coletora $=>$ Programa Fidelidade $=>$ Variedade. Neste caso verifica-se que a presença de Cortesia, Caixa Coletora e Programa Fidelidade (sem Variedade) também não foi suficiente para trazer um alto grau de satisfação aos respondentes (satisfação prevista de 0,61$)$. O grande salto que ocorre na satisfação quando o último atributo obrigatório é introduzido mostra o efeito que esses atributos têm, prejudicando a ação dos atributos atrativos se não estiverem presentes.

\section{CONSIDERAC̣ÕES FINAIS}

Segundo Reichheld e Sasser (1990), atender às necessidades dos clientes é chave para sua satisfação e para o sucesso de uma empresa no longo prazo, pois clientes satisfeitos tendem a repetir o consumo com a empresa e são menos sensíveis a preços. $\mathrm{O}$ atendimento dessas necessidades depende do desempenho do produto ou serviço em seus fação; já um baixo desempenho traz grande insatisfação.

Vários trabalhos mostrando aplicações do Modelo Kano em serviços e produtos têm sido publicados na literatura das áreas de qualidade e marketing. (MATZLER et al., 1996; HUISKONEN; PIRTTILÄ, 1998; ZHANG; DRAN, 2001; TING; CHEN, 2002; WASSENAAR et al., 2003; TONTINI et al., 2004; MATZLER; FUCHS; SCHUBERT, 2004; WALTER; TONTINI; DOMINGUES, 2005; CHEUNG; LEE, 2005; LÖFGREN; WITELL, 2005). Porém, poucos trabalhos têm procurado estudar teoricamente os métodos para identificação dos atributos segundo o Modelo Kano. (PICOLO, 2005; TONTINI; SILVEIRA, 2007; PICOLO; TONTINI, 2006; TONTINI; SANT'ANA, 2007), sendo uma área de pesquisas ainda a ser desenvolvida.

A mera identificação dos atributos como atrativos, obrigatórios ou unidimensionais e sua incorporação no serviço não garantem os efeitos desejados na satisfação dos clientes. Os estudos publicados na literatura identificam a classificação dos atributos segundo o Modelo Kano de forma isolada (KANO et al., 1984; MATZLER et al., 1996; TONTINI et al., 2004; WALTER; TONTINI; DOMINGUES, 2005), ou em conjunto (TING; CHEN, 2002; CHEUNG; LEE, 2005), mas sem estudar o efeito das interações entre eles. O pressuposto desses trabalhos é que as interações entre os atributos são inexistentes ou desprezíveis, assumindo que a satisfação é formada pela adição dos efeitos dos atributos individuais. Porém, seria possível que o efeito dos atributos na satisfação não seja aditivo e nem sua interação desprezível? Caso a resposta seja positiva, isto poderia levar à não identificação de atributos relevantes para a satisfação dos clientes se a interação não for levada em consideração. $\mathrm{O}$ raciocínio básico por traz dessa pergunta pode ser demonstrado por uma outra pergunta: "Será que a falta de limpeza ou cortesia em um restaurante pode ser compensada pela oferta de atributos atrativos como ar condicionado, ambiente 
e cardápios exóticos?”. Apesar de o senso comum levar a se responder negativamente, não foram encontrados trabalhos que estudam este problema de forma científica. O estudo das interações entre os atributos é importante na medida em que interações não identificadas podem levar uma empresa a projetar serviços e/ou produtos que não incorporem atributos relevantes, que apresentam impacto na satisfação somente em conjunto com outros atributos.

Assim, esse trabalho estudou a interação entre atributos atrativos e obrigatórios na formação da satisfação de clientes de videolocadoras. Quatro atributos, presença de Caixa Coletora e Programa Fidelidade (atrativos); Cortesia e Variedade de Filmes (obrigatórios), foram estudados por meio de um delineamento experimental com fatorial completo.

Como conclusão verificou-se que os atributos atrativos, sozinhos, não trazem satisfação se presentes. O mesmo acontece para os atributos obrigatórios. Por outro lado, foi possível verificar que os atributos obrigatórios não apresentaram interação entre si. No estudo aqui realizado o efeito dos atributos obrigatórios não é potencializado pela presença ou ausência de outros atributos obrigatórios. Já os atributos atrativos têm ampliado seu potencial de proporcionarem sa- tisfação aos clientes quando os obrigatórios estão presentes ou têm desempenho suficiente.

Este trabalho apresenta um primeiro passo para se estudar a interação entre atributos atrativos e obrigatórios. Os resultados aqui conseguidos não podem ser generalizados para outros atributos do mesmo serviço ou para outros serviços. Outra possível limitação diz respeito ao uso do delineamento experimental com fatorial completo, o que põe uma sobrecarga nos respondentes e introduz incertezas no resultado. Um possível estudo a ser realizado seria se utilizar dados de pesquisas de satisfação de clientes para ajustar equações lineares de alta ordem, isto é, equações com variáveis independentes que representem a interação entre os atributos, para modelar seu efeito na satisfação geral. Isto reduziria o esforço para responder ao questionário e poderia ampliar o número de atributos estudados.

A implicação gerencial dos resultados deste estudo é que os fornecedores de serviços devem identificar os atributos obrigatórios para o consumidor e mantê-los em um nível adequado, pois somente assim os atributos atrativos, que trazem diferencial no mercado, terão pleno efeito no aumento da satisfação dos clientes.

\section{Artigo recebido em 16/11/2006 Aprovado para publicação em 08/10/2007}

\section{- Referências}

ANDERSON, E. W.; MITTAL, V. Strengthening the satisfaction-profit chain. Journal of Service Research, Maryland, v. 3, n. 2, p. 107-1290, 2000.

BERGER, C.; BLAUTH, R.; BOGER, D.; et al. Kano's methods for understanding customer-defined quality. Center for Quality Management Journal, Massachusetts, v. 2, n. 4, p. 3-35, fall 1993.

DESZCA, G.; MUNRO, H.; NOORI, H. Developing breakthrough products: challenges and options for market assessment, Journal of Operations Management, East Lansing, v. 17, n. 6, p. 613-630, 1999.

GARVER, M. S. Best practices in identifying customer-driven improvement opportunities. Industrial Marketing Management, Vernon, v. 32, n. 6, p. 455-466, 2003.
GIBSON, L. D. What's wrong with conjoint analysis? Marketing Research, v. 13 n. 4, p. 16-20, 2001.

Green, P. E.; Krieger, A. M.; Wind Y. J. Thirty years of conjoint analysis: Reflections and prospects. Interfaces, p. S56-S73, Mai/Jun, 2001

HAIR, J. F. JR.; ANDERSON, R. A.; TATHAM, R. L.; BLACK, W. C. Análise Multivariada de Dados. 5. ed., Porto Alegre: Bookman, 2005.

HUISKONEN, J.; PIRTTILÄ, T. Sharpening logistics customer service strategy planning by applying Kano's quality element classification. International Journal of Production Economics, Linköping, v. 56-57, p. 253-260, sept. 1998.

KANO N.; SERAKU N.; TAKAHASHI F.; TSUJI S. Attractive quality vs must be quality. Journal of the Japanese Society for Quality Control, v. 14, n. 2, p. 39-48, 1984.
LEE, M. K. O.; CHEUNG, C. M. K. The Asymmetric effect of website attribute performance on satisfaction: an empirical study. Proceedings of the 38th Hawaii International Conference on System Sciences, p.10, 2005.

LÖFGREN, M.; WITELL, L. Kano's Theory of Attractive Quality and Packaging. The Quality Management Journal, v. 12, n. 3, p.7, 2005.

MARTILLA, J. A.; JAMES, J. C. Importanceperformance analysis. Journal of Marketing, New York, v. 41, n. 1, p. 77-79, 1977.

MATZLER, K.; HINTERHUBER, H. H.; BAILON, F.; SAUERWEIN, E. How to delight your customers. Journal of Product $\mathcal{E}$ Brand Management, Santa Barbara, v. 5 , n. 2, p. 6-17, mar. 1996.

MATZLER, K.; BAILOM, F.; HINTERHUBER, H.; RENZL, B.; PICHLER, J. The asymme- tric relationship between attribute-level performance and overall customer satisfaction: a reconsideration of the importance-performance analysis. Industria Marketing Management, Vernon, v. 33, n. 4, p. 271-277, may 2004.

MATZLER, K.; FUCHS, M.; SCHUBERT, A K. Employee satisfaction: does Kano's model apply? Total Quality Management, v. 15 , n. 9-10, p. 1179-1198, NovemberDecember, 2004.

MONTGOMERY, D. C. Introdução ao Controle Estatístico da Qualidade. 4. ed. Rio de Janeiro: LTC - Livros Técnicos e Científicos Editora S.A., 2004.

PICOLO, J. D. Influência do desempenho de atributos de produtos ou serviços na satisfação dos clientes: uma análise comparativa entre diferentes técnicas de pesquisa. Dissertação (Mestrado em Programa de Pós Graduação em Administração) - Fundação Universidade Regional de Blumenau. 196 p., 2005. 


\section{Referências}

PICOLO, J. D.; TONTINI, G. Análise do contraste da penalidade e da recompensa (PRC): identificando oportunidades de melhoria em um serviço. In: Encontro da Associação Nacional dos Programas de Pós-Graduação em Administração ENANPAD, 2006, Salvador. Anais do XXX ENANPAD, v. 30, p. 1-16, 2006.

REICHHELD, F. F.; SASSER, W.E. Zerodefections: Quality comes to services. Harvard Business Review, Boston, v. 68, n. 5, p. 105-11, sept./oct., 1990

Sauerwein, E. Experiences with the reliability and validity of the Kanomethod: comparison to alternate forms of classification of product requirements. In: Symposium on QFD, 1999, Michigan. Transactions of the 11th Symposium on QFD, p. 1-14, 1999.
TING, S. C.; CHEN, C. N. The asymmetrical and non-linear effects of store quality attributes on customer satisfaction. Total Quality Management, v. 13, n. 4, p. 547569, 2002.

TONTINI, G. Como identificar atributo atrativos e obrigatórios para o consumidor. Revista de Negócios, Blumenau, v. 8, n. 1, p. 19-28, 2003. Disponível em <http:// www.rn.furb.br>

TONTINI, G.; OSTETTO, F. S. M.; ARRUDA, H. F.; PICCOLO, J. D.; GUARANHA, J. B. Análise de oportunidades de melhoria em laboratórios fotográficos através da integração da matriz de importância $x$ desempenho com o modelo Kano de qualidade. Revista de Negócios, Blumenau, v. 9, n. 3, p. 179-190, 2004. Disponível em <http://www.rn.furb.br>.
TONTINI, G.; SILVEIRA, A. Identification of satisfaction attributes using competitive analysis of the improvement gap. International Journal of Operations \& Production Management, v. 27, n. 5, p. 482-500, 2007.

TONTINI, G.; SANT'ANA, A. J. Identificação de atributos críticos de satisfação em um serviço através da análise competitiva do gap de melhoria. Gestão e Produção, São Carlos, v. 14, n. 1, p. 43-54, jan.abr. 2007.

WALTER, S. A.; TONTINI, G.; DOMINGUES, M. J. C. S. Identificando oportunidades de melhoria em um curso superior através da análise da satisfação dos alunos.
In: Encontro da Associação Nacional dos Programas de Pós-Graduação em Administração - ENANPAD, 2005, Brasília. Anais do XXIX ENANPAD, v. 29, p. 1-15. 2005.

WASSENAAR, H. J.; CHEN, W.; CHENG, J.; SUDJIANTO, A. Enhancing discrete choice demand modeling for decisionbased design. Proceedings of DETC'03, ASME 2003 Design Engineering Technical Conferences and Computers and Information in Engineering Conference. Chicago, Illinois, USA, September 2-6, 2003;

ZHANG, P.; DRAN, G. V. Expectations and rankings of website quality features: results of two studies on user perceptions. Proceedings of the 34th Hawail International Conference on System Sciences. p. 1-10, 2001.

\section{n Sobre os autores}

\section{Gérson Tontini}

Departamento de Administração, Universidade Regional de Blumenau - FURB

End.: Rua Antônio da Veiga, 140 - Blumenau - SC - 89010-971

Tel./Fax: (47) 3321-0285

E-mail: tontini@furb.br.

\section{André José Sant'Ana}

Mestrado em Engenharia Ambiental

Universidade Regional de Blumenau - FURB - Campus II

End.: Rua São Paulo, 3250 - Blumenau - SC - 89030-000

E-mail: andrerj@furb.br. 


\section{ANEXO 1 - QUESTIONÁRIO UTILIZADO, COM DELINEAMENTO EXPERIMENTAL DO TIPO FATORIAL COMPLETO, COM 24 COMBINACÕ̃ES, COM RÉPLICAS.}

Assinale sua satisfação com as seguintes situações:

\begin{tabular}{|c|c|c|c|c|c|c|c|c|c|c|}
\hline \multicolumn{2}{|r|}{ SITUAÇÃO } & \multicolumn{4}{|c|}{$\begin{array}{l}\text { MUITO } \\
\text { INSATISFEITO }\end{array}$} & \multicolumn{3}{|c|}{ NEUTRO } & \multicolumn{2}{|l|}{$\begin{array}{r}\text { MUITO } \\
\text { SATISFEITO }\end{array}$} \\
\hline- & $\begin{array}{l}\text { Tiver grande variedade de filmes para escolher, o atendimento for } \\
\text { cortês e simpático, tiver programa fidelidade, e não tiver caixa } \\
\text { coletora para devolução de filmes fora do horário comercial. }\end{array}$ & -5 & -4 & -3 & -2 & -1 & 0 & +1 & $+2+3+4+$ & +5 \\
\hline ऽ & $\begin{array}{l}\text { Tiver grande variedade de filmes para escolher, o atendimento for } \\
\text { cortês e simpático, não tiver programa fidelidade, e não tiver caixa } \\
\text { coletora para devolução de filmes fora do horário comercial. }\end{array}$ & -5 & -4 & -3 & -2 & -1 & 0 & +1 & $+2+3+4+$ & +5 \\
\hline 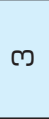 & $\begin{array}{l}\text { Tiver pequena variedade de filmes para escolher, o atendimento for } \\
\text { cortês e simpático, não tiver programa fidelidade, e não tiver caixa } \\
\text { coletora para devolução de filmes fora do horário comercial. }\end{array}$ & -5 & -4 & -3 & -2 & -1 & 0 & +1 & $+2+3+4+$ & +5 \\
\hline$\checkmark$ & $\begin{array}{l}\text { Tiver grande variedade de filmes para escolher, o atendimento não } \\
\text { for cortês e simpático, tiver programa fidelidade e não tiver caixa } \\
\text { coletora para devolução de filmes fora do horário comercial. }\end{array}$ & -5 & -4 & -3 & -2 & -1 & 0 & +1 & $+2+3+4+$ & +5 \\
\hline ما & $\begin{array}{l}\text { Tiver pequena variedade de filmes para escolher, o atendimento não } \\
\text { for cortês e simpático, não tiver programa fidelidade, e tiver caixa } \\
\text { coletora para devolução de filmes fora do horário comercial. }\end{array}$ & -5 & -4 & -3 & -2 & -1 & 0 & +1 & $+2+3+4+$ & +5 \\
\hline$\bullet$ & $\begin{array}{l}\text { Tiver pequena variedade de filmes para escolher, o atendimento } \\
\text { for cortês e simpático, tiver programa fidelidade, e não tiver caixa } \\
\text { coletora para devolução de filmes fora do horário comercial. }\end{array}$ & -5 & -4 & -3 & -2 & -1 & 0 & +1 & $+2+3+4+$ & +5 \\
\hline$\Lambda$ & $\begin{array}{l}\text { Tiver grande variedade de filmes para escolher, o atendimento for } \\
\text { cortês e simpático, não tiver programa fidelidade, e tiver caixa } \\
\text { coletora para devolução de filmes fora do horário comercial. }\end{array}$ & -5 & -4 & -3 & -2 & -1 & 0 & +1 & $+2+3+4+$ & +5 \\
\hline$\infty$ & $\begin{array}{l}\text { Tiver pequena variedade de filmes para escolher, o atendimento } \\
\text { não for cortês e simpático, tiver programa fidelidade, e tiver caixa } \\
\text { coletora para devolução de filmes fora do horário comercial. }\end{array}$ & -5 & -4 & -3 & -2 & -1 & 0 & +1 & $+2+3+4+$ & +5 \\
\hline の & $\begin{array}{l}\text { Tiver grande variedade de filmes para escolher, o atendimento for } \\
\text { cortês e simpático, tiver programa fidelidade, e tiver caixa coletora } \\
\text { para devolução de filmes fora do horário comercial. }\end{array}$ & -5 & -4 & -3 & -2 & -1 & 0 & +1 & $+2+3+4+$ & +5 \\
\hline$\stackrel{\bullet}{\sim}$ & $\begin{array}{l}\text { Tiver grande variedade de filmes para escolher, o atendimento não } \\
\text { for cortês e simpático, tiver programa fidelidade, tiver caixa coletora } \\
\text { para devolução de filmes fora do horário comercial. }\end{array}$ & -5 & -4 & -3 & -2 & -1 & 0 & +1 & $+2+3+4+$ & +5 \\
\hline 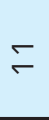 & $\begin{array}{l}\text { Tiver pequena variedade de filmes para escolher, o atendimento não } \\
\text { for cortês e simpático, tiver programa fidelidade, e não tiver caixa } \\
\text { coletora para devolução de filmes fora do horário comercial. }\end{array}$ & -5 & -4 & -3 & -2 & -1 & 0 & +1 & $+2+3+4+$ & +5 \\
\hline$\stackrel{\mathbb{\sim}}{\sim}$ & $\begin{array}{l}\text { Tiver pequena variedade de filmes para escolher, o atendimento } \\
\text { for cortês e simpático, não tiver programa fidelidade, e tiver caixa } \\
\text { coletora para devolução de filmes fora do horário comercial. }\end{array}$ & -5 & -4 & -3 & -2 & -1 & 0 & +1 & $+2+3+4+$ & +5 \\
\hline$\stackrel{m}{\leftarrow}$ & $\begin{array}{l}\text { Tiver grande variedade de filmes para escolher, o atendimento não for } \\
\text { cortês e simpático, não tiver programa fidelidade, e não tiver caixa } \\
\text { coletora para devolução de filmes fora do horário comercial. }\end{array}$ & -5 & -4 & -3 & -2 & -1 & 0 & +1 & $+2+3+4+$ & +5 \\
\hline$\stackrel{ナ}{\leftarrow}$ & $\begin{array}{l}\text { Tiver pequena variedade de filmes para escolher, o atendimento não } \\
\text { for cortês e simpático, não tiver programa fidelidade, e não tiver caixa } \\
\text { coletora para devolução de filmes fora do horário comercial. }\end{array}$ & -5 & -4 & -3 & -2 & -1 & 0 & +1 & $+2+3+4+$ & +5 \\
\hline$\stackrel{\llcorner}{\leftarrow}$ & $\begin{array}{l}\text { Tiver pequena variedade de filmes para escolher, o atendimento for } \\
\text { cortês e simpático, tiver programa fidelidade, e tiver caixa coletora } \\
\text { para devolução de filmes fora do horário comercial. }\end{array}$ & -5 & -4 & -3 & -2 & -1 & 0 & +1 & $+2+3+4+$ & +5 \\
\hline$\stackrel{\bullet}{\leftarrow}$ & $\begin{array}{l}\text { Tiver grande variedade de filmes para escolher, o atendimento não } \\
\text { for cortês e simpático, não tiver programa fidelidade, e tiver caixa } \\
\text { coletora para devolução de filmes fora do horário comercial. }\end{array}$ & -5 & -4 & -3 & -2 & -1 & 0 & +1 & $+2+3+4+$ & \\
\hline
\end{tabular}

Fonte: autores. 